International Journal of Pure and Applied Mathematics

Volume 89 No. 5 2013, 647-657

ISSN: 1311-8080 (printed version); ISSN: 1314-3395 (on-line version)

url: http://www.ijpam.eu

doi: http://dx.doi.org/10.12732/ijpam.v89i5.1

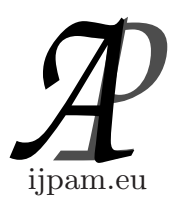

\title{
MATHEMATICAL MODEL OF THREE SPECIES FOOD CHAIN WITH HOLLING TYPE-III FUNCTIONAL RESPONSE
}

\author{
Mada Sanjaya Waryano Sunaryo ${ }^{1}$, Zabidin Salleh ${ }^{2}$, Mustafa Mamat ${ }^{3}$ \\ ${ }^{1}$ Department of Physics \\ Faculty of Science and Technology \\ Universitas Islam Negeri Sunan Gunung Djati \\ Bandung, INDONESIA \\ ${ }^{2,3}$ Department of Mathematics \\ Faculty of Science and Technology \\ Universiti Malaysia Terengganu \\ 21030, Kuala Terengganu, Terengganu, MALAYSIA
}

\begin{abstract}
In this paper, we study ecological model with a tritrophic food chain composed of a classical Lotka-Volterra functional response for prey and predator, and a Holling type-III functional response for predator and superpredator. There are two equilibrium points of the system. In the parameter space, there are passages from instability to stability, which are called Hopf bifurcation points. For the first equilibrium point, it is possible to find bifurcation points analytically and to prove that the system has periodic solutions around these points. Furthermore the dynamical behavior of this models are investigated. The dynamical behavior is found to be very sensitive to parameter values as well as the parameters of the practical life. Computer simulations are carried out to explain the analytical findings.
\end{abstract}

AMS Subject Classification: 34C23, 37G15, 37N25, 70K20,70K50 Key Words: food chain model, Lotka-Volterra model, Holling type-III functional response, Hopf bifurcation

Received: April 6, 2013

(c) 2013 Academic Publications, Ltd. url: www.acadpubl.eu

${ }^{\S}$ Correspondence author 


\section{Introduction}

Interaction networks in natural ecosystems consist of simple units known as food chains. Modeling efforts of the dynamics of food chains initiated long ago confirm that food chains have a very rich dynamics. In the first place, Lotka [9] and Volterra [16] independently developed a simple model of interacting species that still bears their joint names which can be stated as

$$
\begin{aligned}
& \frac{d x}{d t}=a_{1} x-b_{1} x y \\
& \frac{d y}{d t}=-a_{2} y+b_{2} x y
\end{aligned}
$$

with all parameters positive, $a_{1}, a_{2}, b_{1}, b_{2}>0$. Notice that, $x$ is the prey population and $y$ is the predator population, the predator $y$ preys on $x$. The parameters $a_{1}$ is the prey growth rate in the absence of the predators, $b_{1}$ is the capture rate of prey by per predator, $b_{2}$ is the rate at which each predator converts captured prey into predator births and $a_{2}$ is the constant rate at which death in the absence of prey. They showed that ditrophic food chains (i.e. prey-predator systems) permanently oscillate for any initial condition if the prey growth rate is constant and the predator functional response is linear.

Based on experiments, Holling [6, 7, 8, 14, 15] suggested three different kinds of functional responses for different kinds of species to model the phenomena of predator, which resulted the standard Lotka-Volterra system more realistic. Biologically, it is quite natural for the existence and asymptotical stability of equilibria and limit cycles for autonomous predator-prey systems with these functional responses.

Almost each of the food chain models considered in ecological literature are constructed by invoking same type of functional responses for $(x, y)$ and $(y, z)$ populations. But a different selection of functional response would be perhaps more realistic in this context. In 2011, a mathematical model by mixed LotkaVolterra and Holling Type-II functional response was proposed [10]. From this point of view, we have considered and investigated a classical (nonlogistic) Lotka-Volterra functional response for the species $x$ and $y$ and a Holling type-III functional response for the species $y$ and $z$.

\section{The Mathematical Model}

The classical food chain models with only two trophic levels are shown to be insufficient to produce realistic dynamics $[1,2,3,4,10,11,13]$. Therefore, in 
this paper, we consider three species food chain interaction. With non dimensionalization, the system of three-species food chain can be written as

$$
\begin{aligned}
& \frac{d x}{d t}=\left(a_{1}-b_{1} y\right) x ; \\
& \frac{d y}{d t}=\left(-a_{2}+b_{2} x-\frac{c_{1} z y}{D^{2}+y^{2}}\right) y ; \\
& \frac{d z}{d t}=\left(-a_{3}+\frac{c 2 y^{2}}{D^{2}+y^{2}}\right) z
\end{aligned}
$$

where $x, y$, and $z$ denote the non dimensional population density of the prey, predator, and top predator respectively. The predator $y$ preys on $x$ and the predator $z$ preys on $y$. Furthermore, $a_{1}, a_{2}, a_{3}, b_{1}, b_{2}, c_{1}, c_{2}$, and $D$ are the intrinsic growth rate of the prey, the death rate of the predator, the death rate of the top predator, predation rate of the predator, the conversion rate, the maximal growth rate of the predator, conversion factor, and the half saturation constant respectively.

\subsection{Equilibrium Point Analysis}

According to $[5,12,10,11,13]$, the equilibrium points of (2) denoted by $E(\bar{x}, \bar{y}, \bar{z})$, are the zeros of its nonlinear algebraic system which can be written as

$$
\begin{aligned}
\left(a_{1}-b_{1} y\right) x & =0 ; \\
\left(-a_{2}+b_{2} x-\frac{c_{1} y}{D^{2}+y^{2}}\right) y & =0 ; \\
\left(-a_{3}+\frac{c y^{2}}{D^{2}+y^{2}}\right) z & =0 .
\end{aligned}
$$

By considering the positivity of the parameters and the unknowns, we have two positive equilibrium points given by $E_{0}(0,0,0)$, and $E_{1}\left(x_{1}, y_{1}, 0\right)$ with $x_{1}=a_{2} / b_{2}$ and $y_{1}=a_{1} / b_{1}$.

\subsection{Stability of Equilibrium Points}

The dynamical behavior of equilibrium points can be studied by computing the eigenvalues of the jacobian matrix $J$ of system (2) where

$$
J(\bar{x}, \bar{y}, \bar{z})=\left[\begin{array}{ccc}
a_{1}-b_{1} \bar{y} & -b_{1} \bar{x} & 0 \\
b_{2} \bar{y} & -a_{2}+b_{2} \bar{x}-\frac{2 c_{1} y D^{2} \bar{z}}{\left(D^{2}+\bar{y}^{2}\right)^{2}} & -\frac{c_{1} \bar{y}^{2}}{\left(D^{2}+\bar{y}^{2}\right)} \\
0 & \frac{2 c_{2} y D^{2} \bar{z}}{\left(D^{2}+\bar{y}^{2}\right)^{2}} & -a_{3}+\frac{c_{2} \bar{y}^{2}}{\left(D^{2}+\bar{y}^{2}\right)}
\end{array}\right] .
$$

At most, there exists two positive equilibrium points for system (2). The existence and local stability conditions of these equilibrium points are as follows: 
1. The Jacobian matrix (4) at the equilibrium point $E_{0}(0,0,0)$ is

$$
J(0,0,0)=\left[\begin{array}{ccc}
a_{1} & 0 & 0 \\
0 & -a_{2} & 0 \\
0 & 0 & -a_{3}
\end{array}\right] .
$$

The eigenvalues of the Jacobian matrix (5) are $\lambda_{1}=a_{1}, \lambda_{2}=-a_{2}$, and $\lambda_{3}=-a_{3}$. Hence, the equilibrium point $E_{0}$ is a saddle point.

2. The Jacobian matrix (4) at the equilibrium point $E_{1}\left(x_{1}, y_{1}, 0\right)$ is

$$
J\left(x_{1}, y_{1}, 0\right)=\left[\begin{array}{ccc}
0 & -\frac{b_{1} a_{2}}{b_{2}} & 0 \\
\frac{b_{2} a_{1}}{b_{1}} & 0 & -\frac{c_{1} a_{1}^{2}}{b_{1}^{2}\left(D^{2}+\frac{a_{1}^{2}}{b_{1}^{2}}\right)} \\
0 & 0 & -a_{3}+\frac{c_{2} a_{1}^{2}}{b_{1}^{2}\left(D^{2}+\frac{a_{1}^{2}}{b_{1}^{2}}\right)}
\end{array}\right] .
$$

The eigenvalues of the Jacobian matrix (6) are

$$
\begin{aligned}
& \lambda_{1,2}= \pm i \sqrt{a_{1} a_{2}}, \\
& \lambda_{3}=\frac{c_{2} a_{1}^{2}-a_{3} b_{1}^{2} D^{2}-a_{3} a_{1}^{2}}{b_{1}^{2} D^{2}+a_{1}^{2}} .
\end{aligned}
$$

$E_{1}\left(x_{1}, y_{1}, 0\right)$ is locally and asymptotically stable if

$$
a_{3} a_{1}^{2}+a_{3} b_{1}^{2} D^{2}>c_{2} a_{1}^{2}
$$

\subsection{Hopf Bifurcation Point}

When we are interested to study periodic or quasi periodic behavior of a dynamical system, we need to consider the Hopf bifurcation point. The dynamical system generally $[5,12,10,11,13]$ can be written as:

$$
\dot{v}=F(v, \mu)
$$

where

$$
v=v(x, y, z), \quad \mu=\mu\left(a_{1}, a_{2}, a_{3}, b_{1}, b_{2}, c_{1}, c_{2}\right) .
$$

According to $[5,12,10,11,13]$, the system (2) can be written in the form (9) and $(10)$. If an ordered pair $\left(v_{0}, \mu_{0}\right)$ satisfied the following conditions:

1. $F\left(v_{0}, \mu_{0}\right)=0$; 
2. $J(v, \mu)$ has two complex conjugate eigenvalues

$$
\lambda_{1,2}=a(v, \mu) \pm i b(v, \mu)
$$

around $\left(v_{0}, \mu_{0}\right)$;

3. $a\left(v_{0}, \mu_{0}\right)=0, \nabla a\left(v_{0}, \mu_{0}\right) \neq 0$ and $b\left(v_{0}, \mu_{0}\right) \neq 0$;

4. the third eigenvalues $\lambda_{3}\left(v_{0}, \mu_{0}\right) \neq 0$;

then $\left(v_{0}, \mu_{0}\right)$ is called a Hopf bifurcation point.

For the system (2), the equilibrium points $E_{0}(0,0,0)$ and $E_{1}\left(x_{1}, y_{1}, 0\right)$ satisfy the condition $F\left(v_{0}, \mu_{0}\right)=0$, and for the equilibrium point $E_{1}\left(x_{1}, y_{1}, 0\right)$ we have two complex conjugate eigenvalues (7) with the real part of the eigenvalues are zero. The last condition $\lambda_{3}\left(v_{0}, \mu_{0}\right) \neq 0$ is satisfied if

$$
a_{3} a_{1}^{2}+a_{3} b_{1}^{2} D^{2} \neq c_{2} a_{1}^{2} .
$$

The equation (8) and (11) are satisfied if

$$
a_{30}=\frac{a_{1}^{2} c_{2}}{a_{1}^{2}+b_{1}^{2} D^{2}} .
$$

Hence, $E_{1}$ is stable for $a_{3}>a_{30}$ and unstable for $a_{3}<a_{30}$. The point $\left(v_{0}, \mu_{0}\right)$ which corresponds to $a_{3}=a_{30}$ is a Hopf bifurcation point. This Hopf bifurcation states sufficient condition for the existence of periodic solutions. As one parameter is varied, the dynamics of the system change from a stable spiral to a center to unstable spiral (see Table 1).

\section{Numerical Simulation}

Analytical studies always remain incomplete without numerical verification of the results. In this section we present numerical simulation to illustrate the results obtained in previous sections. The numerical experiments are designed to show the dynamical behavior of the system in three main different sets of parameters and initial conditions: $a_{3}<a_{30}, a_{3}=a_{30}$ and $a_{3}>a_{30}$. The coordinates of equilibrium points and the corresponding eigenvalues are can found in Table 1. For showing the dynamics of the system (2) change, the parameter set $\left(a_{1}, a_{2}, b_{1}, b_{2}, c_{1}, c_{2}, D\right)=(0.5,0.5,0.5,0.5,0.6,0.75,10)$ given as a fixed parameters and $a_{3}$ as a varied parameters. The calculation for the parameter set given Hopf bifurcation point $a_{30}=0.007426$ as a reference parameter (12). 
Table 1: Numerical experiment of stability equilibrium point

\begin{tabular}{cccccc}
\hline Case & Parameters & \multicolumn{2}{c}{ Equilibrium Points } & \multicolumn{2}{c}{ Eigenvalues } \\
\cline { 3 - 6 } & & $E_{0}$ & $E_{1}$ & $E_{0}$ & $E_{1}$ \\
\hline$a_{3}<a_{30}$ & $a_{3}=0.001$ & $0,0,0$ & $1,1,0$ & \pm 0.5000 & $\pm 0.5000 i$ \\
& & & & -0.0010 & 0.006426 \\
& $a_{3}=0.005$ & $0,0,0$ & $1,1,0$ & \pm 0.5000 & $\pm 0.5000 i$ \\
& & & & -0.0050 & 0.002426 \\
& $a_{3}=0.0065$ & $0,0,0$ & $1,1,0$ & \pm 0.5000 & $\pm 0.5000 i$ \\
& & & & -0.0065 & 0.000926 \\
$a_{3}=a_{30}$ & $a_{3}=0.007426$ & $0,0,0$ & $1,1,0$ & \pm 0.5000 & $\pm 0.5000 i$ \\
& & & & -0.007426 & 0 \\
$a_{3}>a_{30}$ & $a_{3}=0.008$ & $0,0,0$ & $1,1,0$ & \pm 0.5000 & $\pm 0.5000 i$ \\
& & & & -0.0080 & -0.000574 \\
& $a_{3}=0.01$ & $0,0,0$ & $1,1,0$ & \pm 0.5000 & $\pm 0.5000 i$ \\
& & & & -0.0100 & -0.002574 \\
& $a_{3}=0.05$ & $0,0,0$ & $1,1,0$ & \pm 0.5000 & $\pm 0.5000 i$ \\
& & & & -0.0500 & -0.002574 \\
\hline
\end{tabular}

1. For $a_{3}<a_{30}$.

For the case $a_{3}<a_{30}$ (see Table 1 ) two eigenvalues for $E_{1}$ is pure imaginary initially spiral stability corresponding with center manifold in $x y$ plane and one positive real eigenvalues corresponding with unstable one dimensional invariant curve in $z$ axes. Hence the equilibrium point $E_{1}$ is a locally unstable spiral source and $E_{0}$ is a saddle point with real eigenvalues have opposite sign. In this case the top predator $z$ can survive, growing periodic unstable. On the other hand, prey $x$ and predator $y$ persist and has populations that vary asymptotically stable. The plot of the solution in Figure 1a, 1b, 1c exhibits this behavior.

2. For $a_{3}=a_{30}$.

For the case $a_{3}=a_{30}$ the equilibrium $E_{1}$ has three eigenvalues with zero real part corresponding with stable center point in $x y$ plane (see Table 1). In this case top predator $z$ growth stable periodic. On the other hand, prey $x$ and predator $y$ persist and has populations that vary asymptotically stable. The plot of the solution in Figure 2 exhibits this behavior.

3. For $a_{3}>a_{30}$.

For the case $a_{3}>a_{30}$ (see Table 1) two eigenvalues for $E_{1}$ is pure imag- 

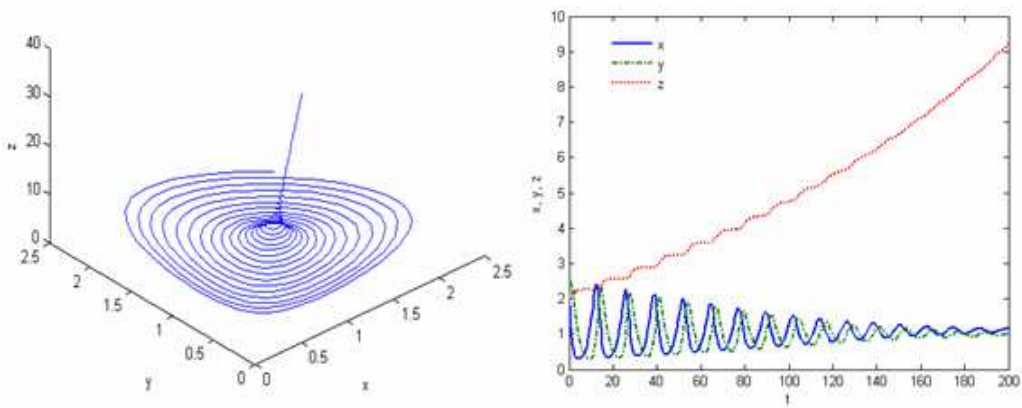

Figure 1a: The solution for $a_{3}<a_{30}$ : phase space and time series for $a_{3}=0.001$.
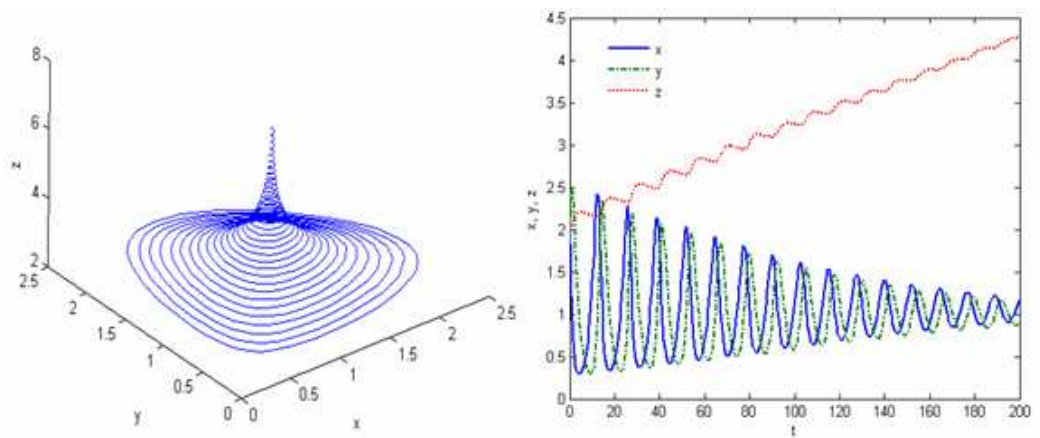

Figure 1b: The solution for $a_{3}<a_{30}$ : phase space and time series for $a_{3}=0.005$.
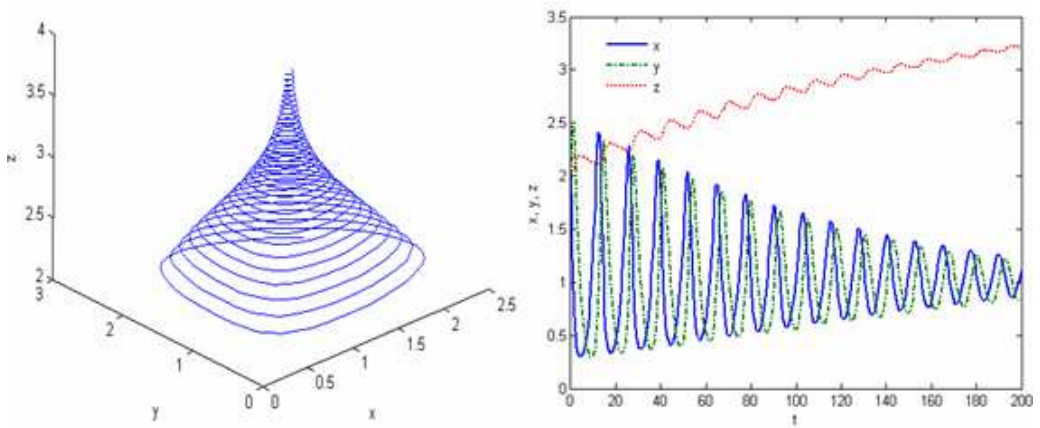

Figure 1c: The solution for $a_{3}<a_{30}$ : phase space and time series for $a_{3}=0.0065$. 

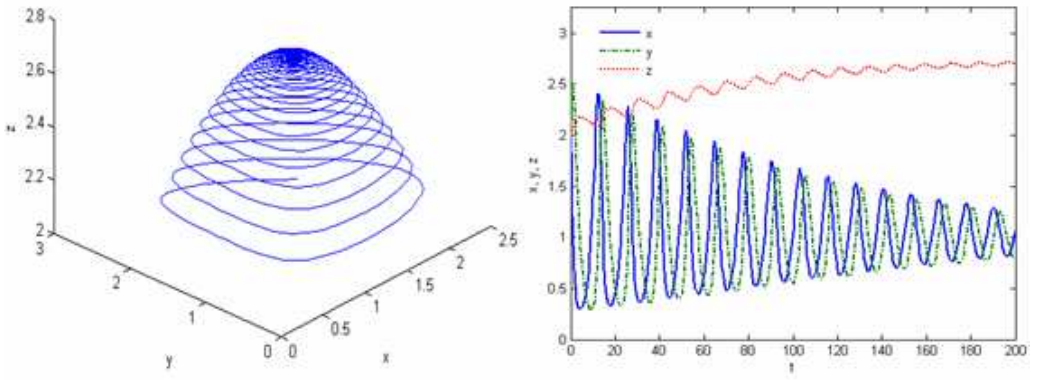

Figure 2: The solution for $a_{3}=a_{30}$ : phase space and time series for $a_{3}=0.007426$.
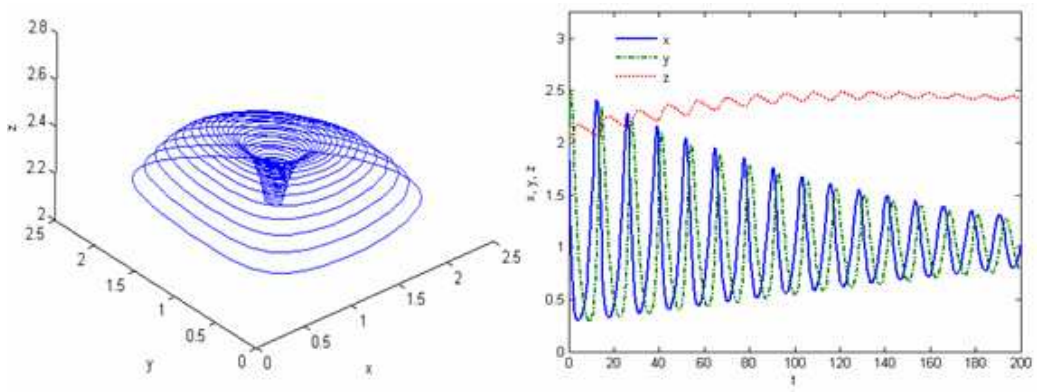

Figure 3a: The solution for $a_{3}>a_{30}$ : phase space and time series for $a_{3}=0.008$.
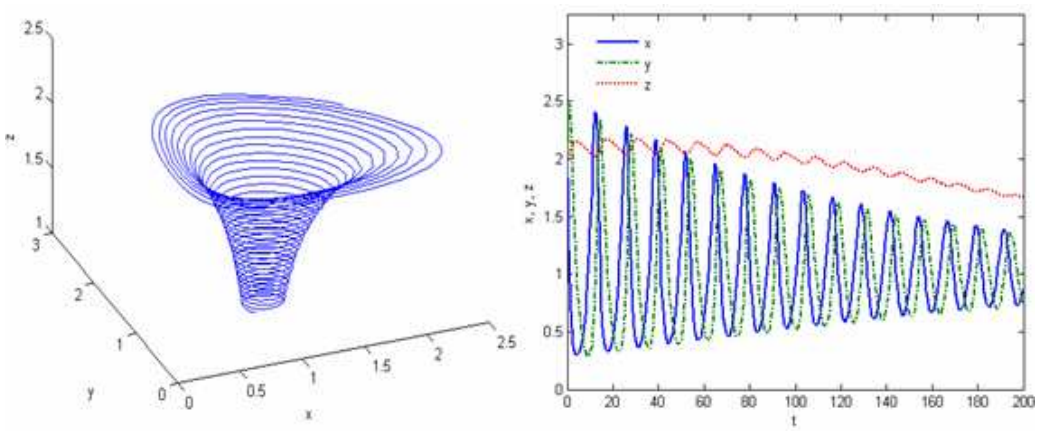

Figure 3b: The solution for $a_{3}>a_{30}$ : phase space and time series for $a_{3}=0.01$. 

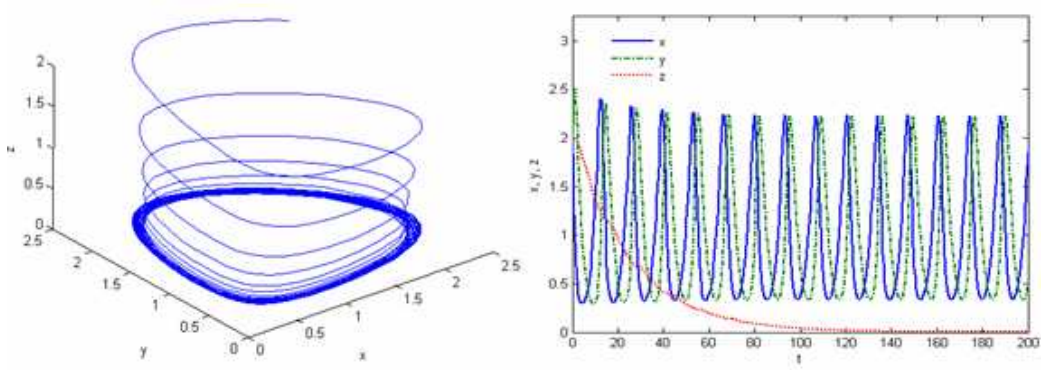

Figure 3c: The solution for $a_{3}>a_{30}$ : phase space and time series for $a_{3}=0.05$.

inary initially spiral stability corresponding with center manifold in $x y$ plane and one negative real eigenvalues corresponding with stable one dimensional invariant curve in $z$ axes. Hence the equilibrium point $E_{1}$ is locally stable spiral sink and $E_{0}$ is a saddle point with real eigenvalues have opposite sign. In this case top predator die. On the other hand, prey $x$ and predator $y$ persist and has populations that vary asymptotically stable. The plot of the solution in Figure 3a, 3b, 3c exhibits this behavior.

\section{Conclusions}

The overall long-term persistence of top species $z$ in (2) hinges solely on the parameters $a_{1}, a_{3}, b_{1}, c_{2}$ and $D$. In particular, if $a_{3}>a_{30}$, then species $z$ decrease over time to dies out, while if $a_{3} \leq a_{30}$, then species $z$ survives. On the other hand species prey $x$ and middle predator $y$ can persist for all condition with asymptotically stable. In this paper, three species food chain model is analyzed and possible dynamical behavior of this system investigated at equilibrium points. It has been shown that, the solutions posses Hopf bifurcations. Both analytically and numerical simulation shown that in certain regions of the parameter space, three species food chain model is sensitively depending on the parameter values. 


\section{Acknowledgments}

This research has beeen partially supported by the Universiti Malaysia Terengganu under the Geran Galakan Penyelidikan (GGP) Ref. No. 68007/2013/80.

\section{References}

[1] B. Dubey and R.K. Upadhyay, Persistence and extinction of one-prey and two-predator system, Nonlinear Analysis, 9, No. 4 (2004), 307-329.

[2] H.I. Freedman and P. Waltman, Mathematical analysis of some three species food-chain models, Math. Biosci., 33, (1977), 257-276. http://dx.doi.org/10.1016/0025-5564(77)90142-0.

[3] A. Hastings and T. Powell, Chaos in a three-species food chain, Ecology, 72, (1991), 896-903. http://dx.doi.org/10.2307/1940591.

[4] A. Hastings and A. Klebanoff, Chaos in three species food chains, J.Math. Biol., 32, (1993), 427-451.

[5] H.C. Hilborn, Chaos and Nonlinear Dynamics, Oxford University Press, New York (1994).

[6] C.S. Holling, The functional response of predator to prey density and its role in mimicry and population regulation, Mem Ent Sec Can., 45, (1965), 1-60. http://dx.doi.org/10.4039/entm9745fv.

[7] S.B. Hsu, T.W. Hwang and Y. Kuang, Global analysis of the MichaelisMenten type ratio-dependence predator-prey system, J. Math. Biol., 42, (2001), 489-506.

[8] S.B. Hsu, T.W. Hwanand Y. Kuang, A ratio-dependent food chain model and its applications to biological control, Math. Biosci., 181, (2003), 55-83.

[9] A.J. Lotka, Elements of Physical Biology, Williams \& Wilkins Co., Baltimore (1925).

[10] W.S. Mada Sanjaya, M. Mamat, Z. Salleh, I. Mohd and N.M. Mohamad Noor, Numerical simulation dynamical model of three species food chain with Holling type-II functional response, Malaysian J. Math. Sci., 5, No. 1 (2011), 1-12. 
[11] M. Mamat, W.S. Mada Sanjaya, Z. Salleh and M.F. Ahmad, Numerical simulation dynamical model of three-species food chain with LotkaVolterra linear functional response, J. Sustain. Sci. Manage., 6, No. 1 (2011), 44-50.

[12] R.M. May, Stability and Complexity in Model Ecosystems, Princeton University Press, Princeton NJ (2001).

[13] Z. Salleh, W.S. Mada Sanjaya, M. Mamat and N.M. Mohamad Noor, The dynamics of a three-species food chain interaction model with MichaelisMenten type functional response, J. Sustain. Sci. Manage., 6, No. 2 (2011), 215-223.

[14] Z. Shuwen and C. Lansun, A Holling II functional response food chain model with impulsive perturbations, Chaos, Solitons \& Fractals, 24, (2005), 1269-1278.

[15] Z. Shuwen and T. Dejun, Permanence in a food chain system with impulsive perturbations, Chaos, Solitons \& Fractals, 40, (2009), 392-400.

[16] V. Volterra, Variazioni e fluttuazioni del numero d'individui in specie animali conviventi, Mem. R. Accad. Naz. dei Lincei., Ser. VI, vol. 2.1, (1926). 
